\title{
Questions éthiques à propos des greffes expérimentales de tissus fotaux humains sur les souris scid/scid
}

\author{
Bruno Cadore, Francis Puech, Jean-Yves Cesbron
}

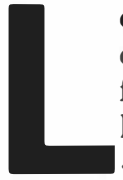

e modèle réalisé par la greffe expérimentale de tissus foetaux sur des souris SCIDhu est considéré comme un "cas-limite" révélateur des questions éthiques posées par le prélèvement de tissus fotaux à fins de recherche. En situant cette recherche dans les cadres éthiques actuels, les auteurs proposent des attitudes pratiques pour la demande de consentement des parents, et de poursuivre la réflexion éthique concernant la justification de ce type de recherche et le problème de l'instrumentalisation de l'humain qu'elle suppose.

\section{Actualité de la question}

La recherche impliquant des tissus fotaux humains est promise à nombre de développements. A côté de l'indispensable formulation de règles déontologiques, l'interrogation éthique fait surgir des questions fondamentales qui méritent d'être portées au débat. La réflexion que nous présentons ici voudrait y contribuer à partir de l'exemple d'une recherche impliquant la greffe expérimentale de tissus fotaux sur des souris scid/scid. Les souris dites SCID (severe combined immune deficiency) présentent une absence d'immunité humorale et cellulaire [1] et tolèrent des xénogreffes [2-4]. Ces souris s'offrent donc à la possibilité de réa$\mathrm{m} / \mathrm{s} n^{\circ} 5$, vol.11, mai 95 liser des modèles expérimentaux permettant des études in vivo par greffes de tissu fotal humain sur l'animal. Une étude des questions éthiques soulevées par ce type de modèle de recherche peut, au premier regard, paraître aborder la question de l'utilisation des tissus foetaux sous un angle trop spécifique. Une expérience de trois ans nous conduit pourtant à penser que, malgré et probablement à cause de son caractère relativement exceptionnel qui en fait une sorte de "cas-limite", ce type de recherche invite à approfondir les enjeux du débat éthique concernant la recherche sur les tissus fotaux.

La récente édition du Dictionnaire permanent de Bioéthique et Biotechnologies propose un article de synthèse sur le thème: "Utilisation des cellules, tissus et produits d'embryons ou de fœetus humains" [5]. Nous nous permettons ici de nous appuyer sur cet article qui présente une synthèse de l'état actuel de la réflexion éthique et surtout juridique à ce propos. Il convient de souligner que cela ne rend compte que du contexte français; nous signalerons au passage, plus loin, les éléments de convergence, voire de divergence, avec d'autres aires culturelles. Il n'y est pas traité explicitement de la question qui fait l'objet précis de la présente réflexion ; cependant, sont très clairement dégagées les grandes questions éthiques actuellement débat- tues concernant l'utilisation des tissus fotaux dans la recherche et la pratique médicales, diagnostique ou thérapeutique. Trois grandes questions d'ordre éthique se dégagent à propos de l'utilisation de tissus fotaux humains en recherche biomédicale: (1) comment se donner les moyens de respecter la dignité de l'humanité du fœetus sur lequel sont prélevés les tissus; (2) comment la finalité de la recherche prend-elle en compte ce souci du respect; (3) quelles sont les conditions définies pour le prélèvement de ces tissus [6, 7] ? C'est par rapport à ces questions que nous avons voulu envisager celles qui sont mises en valeur par le modèle expérimental étudié, le situant ainsi dans le cadre législatif et déontologique déjà existant.

La législation récemment adoptée en France prend position concernant la recherche sur des tissus embryonnaires dans le cadre des techniques d'assistance médicale à la procréation. Cependant, le droit actuel ne donne pas de réponse aussi précise à propos des fœetus après interruption de grossesse. En effet, les fœtus concernés n'ayant jamais été des personnes autonomes vivantes, il n'est pas facile d'appliquer directement à ce propos la loi dite Caillavet (du 22 décembre 1976) concernant les prélèvements sur cadavres humains. Cette ambiguité a conduit le Comité consultatif national d'éthique 
(CCNE) à rendre un certain nombre d'avis [8] à propos des prélèvements sur des embryons ou fœtus morts, en particulier dans l'éventualité d'une possible utilisation thérapeutique sur le mode de la greffe sur le sujet vivant ${ }^{*}$. Le CCNE base son avis princeps de 1984 sur l'affirmation que "l'embryon ou le fœtus doit être reconnu comme une personne humaine potentielle qui est, ou a été, vivante et dont le respect s'impose à tous" (avis $n^{\circ} 1$ ) [8-10]. Les différents avis qui suivent peuvent être ainsi résumés:

- Concernant le cadre de prélèvements pour la recherche, le CCNE souligne: (1) que le but poursuivi doit être d'une particulière importance et apprécié préalablement par un Comité d'éthique qui pourra se donner les moyens d'évaluer le processus de la recherche; (2) la nécessité de garantir l'indépendance entre les deux équipes, obstétricale et de recherche; (3) qu'en aucun cas le but de la recherche ne saurait justifier l'interruption de grossesse $\left(n^{\circ} 2\right)$. - Il insiste de plus sur le fait qu'on ne fera de prélèvement que sur des fœtus n'ayant pas atteint la limite de

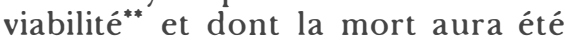
préalablement constatée $\left(n^{\circ} 3\right)$.

- Le consentement des parents est une condition sine qua non du prélèvement, leur donnant la possibilité de s'opposer à de tels prélèvements. - De plus, les pouvoirs publics sont invités à limiter ces pratiques à des établissements faisant preuve de leur compétence et dotés d'un comité d'éthique.

\footnotetext{
* On exclut bien sûr de cette réflexion les questions posées à propos de l'éventualité d'une recherche sur des embryons, par exemple dans le contexte de la pro création artificielle, dont on sait qu'un consensus semble actuellement se dessiner pour réserver cette recherche aux quatorze premiers jours d'existence.

** On fait ici référence aux critères juridiques de viabilité en France (180 jours).

*** Loi $n^{\circ}$ 94-654 du 29 juillet 94 relative au don et à l'utilisation des parties et produits du corps $h u$ main, à l'assitance médicale à la procréation et au diagnostic prénatal (intégrée au Livre VI du Code de
}

- Il ajoute que, si un tel prélèvement de tissus fotaux est considéré comme légitime dans une visée diagnostique, leur usage thérapeutique doit être réservé aux cas exceptionnels et l'utilisation à des fins de recherche doit rester limitée aux situations où l'on poursuit un but spécialement important et ayant comme perspective un progrès thérapeutique. Il exclut toute perspective commerciale.

L'ensemble de ces indications marque les exigences de la réglementation française du respect à l'égard d'une réalité humaine, un fotus, qui, pour être "potentielle", n'en appartient pas moins à l'ordre de l'humain et non à celui de la chose.

C'est au nom de cet impératif du respect de l'humanité qu'un consensus semble se dégager pour appliquer de manière analogique à une recherche portant sur le fotus humain les règles définies pour la recherche impliquant des sujets humains (Loi de décembre 1988). La loi française de juillet $1994^{* * *}$ insiste sur les conditions générales de tout prélèvement: consentement informé, gratuité et anonymat. Dans le cas de "personnes mineures", le consentement express des deux titulaires de l'autorité parentale est requis par écrit, avec une insistance particulière pour les cas de prélèvement pour recherche. Notons que les fins scientifiques de tels prélèvements sont acceptées par la loi adoptée (art. L. 672-4), alors qu'elles étaient exclues dans le projet de loi.

Cette introduction nous permet de situer le cas précis que nous voulons analyser dans un contexte plus global. La particularité de ce modèle expérimental tient donc tout à la fois à ce qu'il s'agit de la greffe de tissu humain sur l'animal, au fait que notre pratique se trouve pour des raisons méthodologiques dans le contexte d'une interruption de grossesse pour raison médicale et que ces implantations sont faites dans un but de recherche immédiatement cognitive. On se trouve donc dans un cas limite où les exigences sont les plus grandes. S'il nous semble pouvoir dire que les instances juridiques françaises de la recherche biomédicale n'interdisent pas ce type d'expérimentation, c'est, bien sûr, sous réser- ve qu'on respecte les exigences portant sur la qualité de sa mise en œuvre. Nous rapportons ici les points sur lesquels un consensus éthique concernant cette recherche s'est établi entre les équipes obstétricales et les chercheurs, ainsi que les problèmes qui restent à débattre.

\section{Les questions spécifiques posées par le modèle utilisé}

\section{La barrière des espèces}

Ce modèle expérimental est habituellement désigné dans la littérature comme une chimère souris/homme [11]. Ce modèle SCID-hu pose donc la question concernant la "construction" d'un organisme-chimère $[12$, 13] animal/humain et de ses enjeux philosophiques [14], rejoignant certaines questions posées à propos des xénotransplantations [15], tout en s'en distinguant puisque ces dernières sont à visée directement thérapeutique. Quoi qu'il en soit de l'analogie des questions posées par les xénotransplantations et de celles qui nous occupent ici du point de vue des relations inter-spécifiques, il s'agit, pour nous, d'examiner la validité d'un modèle d'étude expérimental dont la visée est d'abord cognitive.

La question première est celle de la différence entre ce type de perspective expérimentale et la culture de cellules fœtales à des fins expérimentales, envisageant le problème du rapport que les cellules entretiennent avec un organisme animal non humain unifié dans sa dynamique propre. Existe-t-il, de ce point de vue, un "saut qualitatif" [16] entre le fait de mettre des cellules humaines en situation de se développer à l'intérieur d'un organisme animal, et le fait de cultiver ces cellules en dehors de tout organisme unifié?

On est donc conduit à poser la question de la compatibilité de cette expérimentation avec l'attestation de la dignité inhérente à tout individu appartenant à l'ordre de l'humain. La dignité inaliénable de l'humain s'applique, nous semble-t-il, à tout individu appartenant à cette espèce. A ce titre, le critère de la dignité doit 
impérativement être premier concernant les conditions de prélèvement des tissus. Dans son avis $\mathrm{n}^{\circ} 1$ [8], le CCNE déclare qu' il convient que l'embryon humain ne soit pas considéré comme un matériau de laboratoire". Mais la "dignité " n'est pas, comme telle, applicable aux éléments et produits du corps humain, même si - et, cette fois, au nom de la dignité des humains qui engagent les recherches eux-mêmes - un niveau de respect dans l'action entreprise à partir de ces tissus s'impose [17, 18]. La proposition consiste donc à ne pas attacher la force de l'argument de dignité de la vie humaine à la réalité tissulaire elle-même mais à l'individu humain dont elle provient, en même temps qu'à ceux qui, à son égard, engagent ou autorisent des manipulations et des recherches. A ce titre, elle se situe dans l'ordre symbolique plus que strictement biologique ou ontologique [19]. Les limites assignées à l'expérience peuvent viser à maintenir ces expérimentations comme prolongement d'une étude de culture de cellules in vivo, impliquant donc le maintien de la "cohabitation" de tissus humains en un organisme animal dans les limites temporelles strictement nécessaires à l'observation, excluant la reproduction des animaux, et l'exclusion de tout usage de tissus germinaux humain.

\section{Les conditions du prélèvement : des questions générales}

D'une manière générale, l'une des questions difficiles des recherches sur le tissu foetal est celle des divers types de fotus qui pourraient être utilisés pour de telles expérimentations [20], et des modalités d'obtention du consentement. On écarte bien sûr toute éventualité de "procréation" (a fortiori "production") de fœtus en vue de dons d'organes ou à des fins expérimentales [21, 22] . Le modèle que nous utilisons requiert le prélèvement de tissu thymique à un stade suffisant de développement (vers la $18^{\mathrm{e}}$ semaine d'aménorrhée environ), et ne nous place donc pas dans le cadre des IVG. Si, pour d'autres modèles, cette éventualité devait se présenter, il fau- drait alors envisager si ce contexte particulier d'un choix de convenance modifie les éléments pour obtenir un consentement autonome des parents et si, plus généralement, on peut ou non avoir recours à du tissu provenant de fotus avortés pour des raisons "électives" ".

D'autres s'interrogeront, cependant, sur le fait de prélever des tissus sur des fœetus avortés pour des raisons médicales, au nom du risque, précisément, que la perspective de recherche ne vienne contribuer à justifier trop vite un geste par une perspective "positive». Dans le but d'éviter au maximum l'ambiguité et tout type de pression, on insistera sur le caractère de gratuité du consentement au prélèvement, l'exigence de non-collusion $^{* *}$ entre le choix du couple parental et le projet de la recherche, la dissociation entre l'équipe obstétricale et celle de recherche pour récuser toute collusion entre elles. Cependant, compte tenu de la coordination nécessaire pour la rigueur de la recherche entre l'équipe d'obstétrique et celle du prélèvement et de la recherche proprement dite, il paraît indispensable qu'un lien soit établi entre eux, y compris dans l'intéressement à l'exploitation des résultats du travail. Une évaluation régulière sera donc nécessaire pour permettre de mesurer l'objectivité des choix réalisés et le respect de la non-collusion.

Les règles générales concernant l'usage de tissus fotaux à des fins de recherche témoignent de la volonté des sociétés d'attester l'intangible dignité de l'espèce humaine. Ce souci est particulièrement prégnant lorsque, devant solliciter le consentement des parents pour effectuer la

Le point sur lequel on insiste à ce propos est le suivant: éviter que des décisions d'interruption volontaire de grossesse ne soient motivées, médiatement ou non, par la perspective de recherche intégrant l'usage de tissus fataux. De ce point de vue, il apparait plus facile d'éviter la collusion en prélevant sur des fatus avortés pour raison médicale.

** Loi $N^{\circ} 76-1181$ du 22.12.1976 relative aux prélèvements d'organes. J.O. du 23 décembre 1976 On constate que ces exigences pratiques rejoignent celles de l'Avis du CCNE. greffe de tissu foetal sur l'animal à des fins scientifiques, et en raison des réactions que peut provoquer un tel "passage de frontière", les équipes cherchent à préciser les conditions les meilleures pour cette démarche. Comment atteindre un bon niveau de transparence de l'information en vue du consentement?

Les modalités de cette demande, qui n'est pas seulement celle d'autorisation d'une autopsie, ni celle envisageant la culture de cellules foetales, sont difficiles à définir, puisque les parents doivent y répondre de la manière la plus éclairée et autonome possible. Or, le contexte de l'interruption de la grossesse met le couple dans des conditions d'exercice de son autonomie tout à fait particulières. C'est dans le souci du plus grand respect des couples, vivant le drame de la découverte d'une anomalie, qu'il s'agit de demander ces prélèvements.

Notre pratique nous a conduits à adopter une démarche progressive adaptée à chaque cas. La première condition est, bien sûr, que le protocole de l'expérimentation n'entrave ni la démarche, ni la programmation, ni le déroulement de l'interruption médicale de grossesse. Pour respecter les parents dans leur autonomie et dans l'épreuve qu'ils traversent, le praticien hospitalier évoque l'éventualité d'un prélèvement au cours de l'autopsie (qui, dans ces cas d'interruption médicale de grossesse, est systématiquement pratiquée, sauf refus des parents), au moment où le geste d'interruption de la grossesse est envisagé, cherchant ainsi à préparer le couple à entendre la demande qui sera faite. Ce n'est qu'après l'interruption que le praticien demandera l'accord explicite pour que des tissus fotaux soient prélevés à des fins de recherche de type expérimentale, à visée thérapeutique, en précisant le cadre des travaux (SIDA dans notre cas), sans décrire de façon précise l'ensemble des modalités opératoires ni d'emblée parler de greffes de tissus sur l'animal. Cependant, le dialogue au cours duquel cette information est donnée s'efforce d'être suffisamment ouvert pour que les parents puissent demander davanta- 
ge de précisions. Dans notre expérience, le refus de ces prélèvements concerne environ $30 \%$ des interruptions médicales de grossesse.

Cette adaptation progressive de l'information manif este le souci que les conditions du consentement soient claires sans que ses modalités formelles soient trop lourdes pour les parents. Cette attitude veut donc éviter, d'un côté, la non-information au nom de l'intérêt de la recherche et, de l'autre côté, une information complètement détaillée qui pourrait provoquer un "choc symbolique" chez les parents. Cette attitude peut paraître hypothéquer le discours habituellement tenu sur les exigences du consentement éclairé des personnes. C'est pour cela que nous proposons: (1) d'affirmer préférable de s'exposer à un refus de la part des parents, plutôt que de ne pas être prêt à donner toute l'information; (2) qu'une psychologue et l'équipe des praticiens s'assurent de la qualité du consentement recueilli par une démarche d'évaluation qui a trois objectifs: (a) faire le récit $a$ posteriori des demandes de consentements pour s'assurer que les conditions de l'autonomie des parents comme des soignants étaient suffisamment réunies; (b) vérifier que le "vécu psychologique" du couple et de l'équipe assure une certaine objectivité dans le discernement de la situation; (c) assurer le suivi du couple ayant consenti; (3) finalement, informer suffisamment sur l'intérêt de ce type de modèle et les conditions strictes de son usage, puisqu'on peut prévoir l'extension de ce dernier dans la recherche, comme, par exemple, à propos de l'infection par les VIH [2327].

Cette exigence s'ajoute, bien sûr, à celle d'une évaluation des conditions scientifiques de l'expérimentation.

\section{Exigences pour le discernement}

Il nous semble opportun de souligner les trois axes principaux structurant la réflexion éthique qui est développée à partir de nouveaux champs de recherche biomédicale: (1) la possibilité d'une nouvelle méthode le à justifier cette recherche; (2) les conséquences de son déploiement doivent être envisagées par anticipation; (3) la question du rapport de l'homme à lui-même reste sans cesse posée à nouveaux frais, à la mesure de la performance de la recherche sur l'humain.

\section{Justification de la recherche}

A côté de la qualité du respect qui est manifesté au fœtus au titre de sa nature de "personne humaine potentielle ${ }^{*}[10,28]$, de l'importance du lien parental, de la qualité du type de consentement recueilli et des conditions de liberté et de gratuité qui doivent le caractériser, l'argumentation du bien-fondé scientifique de la recherche entreprise doit être solidement étayée.

Cependant, les problèmes posés par la réalisation de souris SCID-hu ne sont peut-être pas formulés de la façon la plus pertinente à partir de ces axes puisque, dans le cadre général, il s'agit le plus souvent de réflexions menées dans le contexte d'une recherche thérapeutique directe pour un patient malade. Nous nous situons ici dans la perspective immédiate d'une recherche cognitive qui doit démontrer qu'elle a aussi une visée thérapeutique, même si cette dernière est indirecte et incertaine.

Parmi les distinctions utiles pour ce discernement, il convient donc de chercher à hiérarchiser les justifications d'un processus de recherche. Toute recherche ne justifie probablement pas la réalisation d'un tel modèle expérimental. Il est important de distinguer les recherches pour lesquelles ce modèle est le seul moyen d'aboutir et celles qui pourraient être menées avec d'autres types de moyens, fussent-ils moins "séduisants" intellectuellement. Pour honorer cette remarque, il paraît important que toute équipe

* On fait ici aussi référence à la définition proposée par le CCNE en France, qui a élaboré ce "concept" de personne humaine potentielle pour tenter de dire un statut philosophique de l'embryon et du foetus humain. envisageant un tel modèle de recherche se sente tenue de démontrer méthodologiquement qu'il s'agit bien du seul moyen praticable pour la visée poursuivie. Il convient, en particulier, de vérifier que l'affection à l'origine de l'interruption médicale de grossesse, et les conditions de prélèvements, n'obèrent pas la validité du modèle.

Ce problème de la pertinence d'une innovation dans les méthodes scientifiques et celui de la rigueur de leur indication doivent être posés avec d'autant plus d'insistance qu'on peut prévoir un développement dans l'usage des modèles SCID-hu et que l'urgence des questions que l'on cherche à résoudre (celles du SIDA) s'impose aux yeux de beaucoup.

\section{Une recherche à quelle échelle ?}

L'usage de tels modèles pourrait se développer, comme, par exemple, dans le cadre de recherches sur l'infection par le VIH [23-28], mais il convient d'être vigilant pour éviter les risques de certains dérapages provoqués par des enjeux économiques trop puissants. L'expérimentation thérapeutique sur de tels modèles paraît ouvrir la voie à des enjeux financiers si importants que les entreprises pharmaceutiques pourraient souhaiter développer sur une large échelle de tels modèles expérimentaux. Se poserait alors la question de la nécessité de "collecter" en quantité suffisante des tissus fœtaux, ce qui pourrait ouvrir la voie à la "recherche" de tissus disponibles.

Aussi, outre la distinction qu'il est possible d'établir selon l'échelle à laquelle se développent les projets, cela conduit de plus à une réflexion concernant les implications entrepreneuriales et financières. Autant le point de vue économique est désormais souvent impliqué dans le questionnement éthique de la médecine, autant il l'est encore assez peu concernant la recherche. Le type de projet que nous analysons ici oblige pourtant à considérer cette dimension puisque c'est dans ce cadre que se poseront des problèmes touchant à la production pharmaceutique, la brevetisation de modèles animaux, et les bénéfices commerciaux de 
recherches ayant impliqué une phase expérimentale sur des produits humains*. On doit donc considérer les questions concernant la définition des priorités de la recherche et leur interaction avec les systèmes économiques. Ce point souligne la nécessité de la plus grande transparence possible dans le processus de la recherche, de façon à garantir au maximum le respect des règles de qualité et d'évaluation de la recherche. La portée symbolique de tels modèles exige une grande prudence quant aux conditions économiques de leur usage. Il serait souhaitable, par exemple, de proposer des conditions d'accompagnement des entreprises engagées, autorisant par exemple un contrôle des réinvestissements des bénéfices réalisés selon l'argument de la justice ${ }^{* *}$.

\section{L'instrumentalisation de l'humain}

Nous avons souligné que l'utilisation du tissu fœtal a une finalité indirectement orientée vers le bénéfice de l'homme et de sa santé [29], présentant un anthropocentrisme évident. Certains pourraient le contester, mais il se présente aussi à la réflexion comme un critère d'évaluation du bien-fondé de l'entreprise: en quoi telle ou telle pratique peut-elle être objectivement un bien pour l'humanité ? Implicitement cette question donne une finalité thérapeutique à toute recherche instrumentalisant un élément du corps humain.

\footnotetext{
* Au moins trois brevets ont été déposés. "Determination of efficacy of drugs or vaccine in immunocompromised hosts by administrating xenogeneic tis sue to host before administration of pathogen and drug" J.M. McCune and E.S. Mocarski, 1990, "Growing primordium in immuno-deficient hosts by implanting primordium into immuno-deficient xenogeneic host at site capable of vascularisation, and growing host to give more mature organ ", B. Peault 1991; "Assay for human T-lymphocyte progenitor cells by using test cells to repopulate lym phoid depleted thymus tissue, then implantation into mice and detecting donor lymphocytes ", B. Peault, 1990.
}

** On fait ici référence à l'exigence de la justice dans l'éthique biomédicale, l'un des critères d'évaluation au même titre que l'autonomie ou la "beneficience". Il s'agit de s'assurer que les bénéfices d'une recherche ne sont pas facteurs d'exclusion.

$\mathrm{m} / \mathrm{s} n^{\circ} 5$, vol. 11 , mai 95
Outre les remarques que nous avons faites concernant la dimension symbolique du modèle SCID-hu, il reste à envisager les limites que l'on peut assigner aux projets de développement des connaissances au nom du respect de l'humain. Le critère important qu'il s'agirait de faire valoir est celui des limites dans lesquelles l'homme s'autorise à instrumentaliser les éléments de sa corporéité. L'évolution contemporaine de la science biomédicale est, sans nul doute, orientée de telle sorte que le corps humain subisse un certain degré d'objectivation [30, 31]. La disponibilité du tissu fotal humain le rend particulièrement vulnérable, imposant une prudence qui, dans le cadre de notre expérience, a été à l'initiative de cette réflexion.

\section{Conclusion}

Certes, les débats sont déjà nombreux concernant la recherche sur l'embryon. Leur enjeu est d'inscrire l'exigence du respect de l'humanité au cœur des innovations scientifiques et techniques. L'analyse des questions posées par ce modèle expérimental particulier souligne qu'une pratique de recherche met inévitablement en jeu la conception de l'humain que l'on veut promouvoir.

La recherche fondamentale contraint ainsi la réflexion éthique à approfondir ses fondements anthropologiques, dialogue et démarche indispensables à la formulation de repères pratiques pour évaluer les risques et les chances de cette "instrumentalisation" de l'humain. Cela appelle en même temps au développement d'une certaine cohérence dans les déterminations morales d'une société qui, au-delà des seules limites de la recherche biomédicale, chercherait à faire toujours plus valoir l'exigence du respect de tout être humain, jamais considéré seulement comme un moyen mais toujours aussi comme une fin. C'est en effet dans cette perspective que des critères pratiques pour l'action de recherche trouveraient leur sens. C'est dire l'importance de la contribution des chercheurs, par leur souci éthique, à la qualité d'une société humaine

\section{RÉFÉRENCES}

1. Bosma GC, Custer RP, Bosma MJ. A severe combined immunodeficiency mutation in the mouse. Nature $1983 ; 301: 527-30$.

2. McCune JM, Namikawa R, Kaneshima $H$, Shultz LD, Lieberman M, Weissman IL. The SCID-hu mouse: murine model for the analysis of human hematolymphoid differentiation and function. Science $1988 ; 241: 1632-9$

3. Mosier DE, Gulizia RJ, Baird SM, Wilson DB. Transfer of a functional human immune system to mice with severe combined immunodeficiency. Nature 1988 ; 335 : 256-9.

4. Ware CF, Donato NJ, Dorshkind K. Human, rat or mouse hybridomas secrete high levels of monoclonal antibodies following transplantation into mice with severe combined immunodeficiency disease (SCID). $J$ Immunol Methods 1985; 85 : 353-61.

5. Dictionnaire permanent de Bioéthique et Biotechnologies. Paris: Ed. Législatives, 1994

6. Greely HT. The ethical use of human fetal tissue in medicine. $N$ Engl J Med 1989; 320: $1093-6$

7. Jagiello GM. La science et la société face aux recherches portant sur du matériel foetal d'origine humaine. Gyn Int 1993; 2: 139-47.

8. Comité Consultatif National d'Éthique pour les Sciences de la vie et de la Santé Avis ${ }^{\circ} 1,8,16$ et 23 . Les Avis de 1983 à 1993. Paris : CCNE, 1993.

9. Verspieren P. Le prélèvement de tissus

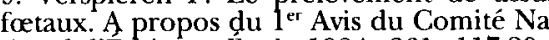
tional d'Ethique. Études 1984; 361 : 117-20.

10. Sève L. La Recherche biomédicale et respect de la personne humaine. Paris: La Documentation Française, 1988.

11. Le Nouveau Petit Robert. Paris: Dictionnaire Le Robert, 1994.

19. Delsol M. Les chimères chez les êtres vivants. In: Berenard J, Bessit M, Debru C. eds. Soi et non soi. Paris: Seuil, 1990: 77-87.

13. Salmon $M$. Les chimères biologiques chez l'homme. In: Berenard J, Bessit $M$ Debru C, eds. Soi et non soi. Paris: Seuil, 1990: 89-102

14. Macer D, Balk RA, Freedman B, Goulet MC. New Creations? Hastings Cent Rep 1991 $32-5$.

15. Drugan A, Evans W, Evans MI. Fetal or gan and xenograft transplantations. Am $J$ Obst Gynecol 1989; 3 : 289-93.

16. Fishman RE. Patenting human being do sub-human creature deserve constitutional protection. Am J Law Med 1989; 15 : 461 82.

17. Nolan K. Genug ist genug: A fetus is not a kidney. Hastings Cent Rep 1988; 18: 13-9. 


\section{RÉFÉRENCES}

18. Robertson J. Rights and public policy in fetal tissue transplants. Hastings Cent Rep $1988 ; 18: 1386-8$.

19. Boné É. Les greffes intracérébrales de tissu foetal. Une nouvelle aporie éthique RTL 1990; 21 : 311-28.

20. Kearney W, Vawter DE, Gervais KG. Fetal tissue research and the misread compromise. Hastings Cent Rep 1991 ; 21 : 7-12.

21. Clark RD, Fletcher J, Petersen G Conceiving a fetus for bone-marrow donation : an ethical problem in prenatal diagnosis. Pren Diag 1989; 9 : 329-34.

22. Strong C. Fetal tissue transplantation: can it be morally insulated from abortion ? J Med Eth 1991 ; 17 : 70-6.

23. Cesbron JY, Agut H, Gosselin B, Candotti D, Raphael M, Puech F, Grandadam M Debre P, Capron A, Autran B. SCID-hu mouse as a model for human lung HIV-1 infection. CR Acad Sci Ser III 1994; 317: 669 74.

24. Kaneshima $H$, Shih CC, Namikawa $R$ Rabin L, Outzen H, Machado SG, McCune JM. Human immunodeficiency virus infection of human lymph nodes in the SCID-hu mouse. Proc Natl Acad Sci USA 1991; 88 4523-7.

25. McCune JM, Namikawa R, Shih CC, Rabin L, Kaneshima H. Suppression of HIV infection in AZT-treated SCID-hu mice. Science $1990 ; 247$ : 564-6.

26. Sandstrom E, Oberg B. Antiviral therapy in human immunodeficiency virus infections : current status 2. Drugs 1993; $45: 637$ 53.

27. Shih CC, Kaneshima H, Rabin L, Namikawa R, Sager P, McGowan J, McCune JM. Postexposure prophylaxis with zidovudine suppresses human immunodeficiency virus type 1 infection in SCID-hu mice in a timedependent manner. I Infect Dis 1991; 163 : 625-7.

28. Jeckner NS. The moral status of patients who are non strict persons. J Clin Ethics $1990 ; 1: 35-8$.

29. Caplan AL. Should fetuses or infants be utilized as organ donors? Bioethics 1987; 1 : $119-40$.

30. The Danish Council of Ethics. Protection of human fetuses. Copenhagen, 1990.

31. Robertson JA. Extracorporeal embryos and the abortion debate. $I$ Contemp Health Policy $1986 ; 2$ : 53-70.

\section{TRRÉS À PART}

\section{Summary}

Ethics and fetal tissue experimental grafts on scid/scid mice

During the last 5 years, C.B.17 scid/scid mice have proven to be outstanding hosts for the propagation of human xenografts. For example, SCID mice reconstituted with fetal lymphoid tissues can be infected with HIV and the experimental results obtained from these chimeric human/mice animals appear to be relevant for human disease and immune function. This article deals with the ethical issues raised by these experimental models. The authors examined the criteria of the fetal tissue process, the modalities to obtain the parents' consent, the scientific justifications needed both to make use of these models and their possible extension.

Remerciements

Nous remercions ceux qui ont bien voulu relire ce texte à ses phases successives d'élaboration: M. le Pr J.-F. Malherbe (Faculté de Théologie de Sherbrooke, Canada), M. Lucien Sève, et MM. les $\operatorname{Pr} B$. Autran, H. Agut et M. Raphaēl (Cnrs URA 625, hôpital Pitié-Salpêtrière, Paris) qui collaborent au travail de laboratoire sur les souris SCID-Hu, programme financé en partie par l'ANRS Contrat $N^{\circ} 3229$.

\section{Bruno Cadore}

Professeur agrégé, centre d'éthique médicale de l'université catholique de Lille, faculté libre de médecine, 60, boulevard Vauban, BP 109, 59046 Lille Cedex, France.

\section{Francis Puech}

Professeur, université de Lille-II, service de pathologie maternelle et foetale, maternité Salengro, 12, rue Malpart, 59800 Lille, France.

\section{Jean-Yves Cesbron}

Maître de conférences, praticien hospitalier, Inserm U. 415, Institut Pasteur de Lille, 1, rue du Professeur Calmette, BP 245, 59019 Lille Cedex, France. 